PROCEEDINGS OF THE

AMERICAN MATHEMATICAL SOCIETY

Volume 128, Number 12, Pages 3505-3511

S 0002-9939(00)05595-7

Article electronically published on May 18, 2000

\title{
ISOMETRICAL EMBEDDINGS OF SEPARABLE BANACH SPACES INTO THE SET OF NOWHERE APPROXIMATIVELY DIFFERENTIABLE AND NOWHERE HÖLDER FUNCTIONS
}

\author{
STANISLAV HENCL
}

(Communicated by Dale Alspach)

\begin{abstract}
The well-known Banach-Mazur theorem says that every separable Banach space can be isometrically embedded into $C([0,1])$. We prove that this embedding can have the property that the image of each nonzero element is a nowhere approximatively differentiable and nowhere Hölder function. It improves a recent result of L. Rodriguez-Piazza where the images are nowhere differentiable functions.
\end{abstract}

\section{INTRODUCTION}

The well-known Banach-Mazur theorem says that every separable Banach space can be isometrically embedded into $C([0,1])$. We prove that this embedding can have the property that the image of each nonzero element is a nowhere approximatively differentiable and nowhere Hölder function. It improves a recent result of L.Rodriguez-Piazza 2 where the images are nowhere differentiable functions (for references about results concerning embeddings into subsets of $C([0,1])$ see [2]). The basic idea of our proof is the same as in [2] but we use a more complicated construction which uses an idea of Malý and Zajíček [1].

Let $\Delta$ be the Cantor set. It is well known that every separable Banach space is isometric to a subspace of $C(\Delta)$ so the following theorem will be enough to obtain the announced result.

Theorem 1. There exist a closed subset $K$ of $[0,1]$ homeomorphic to the Cantor set $\Delta$ and a linear operator $F: C(K) \rightarrow C([0,1])$ such that for every $f \in C(K) \backslash\{0\}$ we have:

(i) $F f(t)=f(t)$ for every $t \in K$, so $F f$ is a continuous extension of $f$ to the whole interval.

(ii) $\|f\|_{\infty}=\|F f\|_{\infty}$, so $F f$ is an isometry.

(iii) $F f$ is nowhere approximatively differentiable and nowhere Hölder function.

In fact we will prove a stronger result:

Proposition 2. Let $\varphi:[0, \infty) \rightarrow[0, \infty)$ be a continuous increasing function such that $\varphi(0)=0$. Then there exist a closed subset $K$ of $[0,1]$ homeomorphic to the Cantor set $\Delta$ and a linear operator $F: C(K) \rightarrow C([0,1])$ such that for every $f \in C(K) \backslash\{0\}$ we have:

Received by the editors January 22, 1999.

1991 Mathematics Subject Classification. Primary 26A27, 46B04.

(C)2000 American Mathematical Society 
(i) $F f(t)=f(t)$ for every $t \in K$.

(ii) $\|f\|_{\infty}=\|F f\|_{\infty}$.

(iii) For all $n \in \mathbf{N}$ it holds that the set $D_{z}=\left\{y \in[0,1]:\left|\frac{F f(z)-F f(y)}{\varphi(|z-y|)}\right|>n\right\}$ has the symmetric upper density 1 at $z$ for all $z \in(0,1)$, the set $D_{0}$ has the right upper density 1 at point 0 and the set $D_{1}$ has the left upper density 1 at point 1 .

Remark. Proposition 2 not only gives us that $F f$ does not have finite approximate derivative but also that $F f$ does not have a finite one-sided preponderant derivative as well (for definition of the preponderant derivative see [3, pages 112-113]).

As a by-product of our construction we will obtain:

Proposition 3. There exists a nonempty perfect set $K \subset[0,1]$ such that every continuous function on $K$ can be extended to $[0,1]$ such that every point $z \in[0,1]$ is a Jarnik point of the extension.

Recall definitions of some notions used above. Suppose that $f$ is measurable on $[0,1]$.

Let $x \in[0,1]$ and $r \in \overline{\mathbf{R}}$. We say that ap- $\lim _{y \rightarrow x} f=r$, if for each neighborhood $U$ of $r$

$$
\lim _{h \rightarrow 0+} \frac{|\{y \in[x-h, x+h] \cap[0,1]: f(y) \in U\}|}{|[x-h, x+h] \cap[0,1]|}=1,
$$

where $|M|$ denotes the Lebesgue measure on $\mathbf{R}$. The function $f$ is said to be approximatively differentiable at a point $x \in[0,1]$ if there exists $L \in \mathbf{R}$ such that

$$
\operatorname{ap}_{-\lim _{y \rightarrow x}} \frac{f(y)-f(x)}{y-x}=L .
$$

A point $x \in[0,1]$ is a Jarník point of $f$ if

$$
\operatorname{ap}_{-\lim _{y \rightarrow x}}\left|\frac{f(y)-f(x)}{y-x}\right|=\infty .
$$

We say that $f$ on $[0,1]$ is a nowhere Hölder function if for all $x \in[0,1]$ and $\alpha>0$

$$
\sup _{y \in[0,1]} \frac{|f(x)-f(y)|}{|x-y|^{\alpha}}=\infty
$$

holds.

If we apply Proposition 2 for $\varphi(t)=-\frac{1}{\ln t}$ we get that property (iii) of Proposition 2 clearly implies property (iii) of Theorem 1 . Thus we devote the rest of the paper to the proofs of Proposition 2 and Proposition 3.

\section{Construction of USEFul SEQuences}

Let $\varphi$ be as in Proposition 2. Put $\psi=\sqrt{\varphi}$. We can clearly find a sequence $\left\{a_{n}\right\}_{n=1}^{\infty}$ such that $1>a_{n}>0$,

$$
\begin{aligned}
& \sum_{j=n+1}^{\infty} a_{j} \leq \frac{a_{n}}{2 n}, \\
& \sum_{j=n}^{\infty} a_{j} \leq \frac{1}{2^{n}} .
\end{aligned}
$$


Further define inductively a sequence $\left\{p_{n}\right\}_{n=1}^{\infty}$ such that $p_{n}>0$,

Put

$$
\begin{aligned}
& p_{1}<\frac{1}{10}, \\
& 2 \psi\left((n-1) p_{n-1}\right) \leq \frac{a_{n}}{n} \text { for } n=2,3, \ldots, \\
& 2 \pi p_{n} \sum_{j=1}^{n-1} \frac{a_{j}}{p_{j}} \leq \frac{a_{n}}{n} \quad \text { for } n=2,3, \ldots, \\
& a_{n} \sqrt{n-1} p_{n-1}>10 n p_{n} \text { for } n=2,3, \ldots, \\
& n p_{n} \searrow 0 .
\end{aligned}
$$

$$
\lambda_{n}=\sqrt{n} p_{n} \quad \text { for } n \in \mathbf{N} .
$$

\section{Construction of $K$}

Define $I_{0,1}=[0,1]$ and $\lambda_{0}=1$. For every $n \in \mathbf{N}$ we will choose $2^{n}$ pairwise disjoint closed intervals $\left\{I_{n, j}\right\}_{j=1}^{2^{n}}$ in $[0,1], I_{n, j}=\left[c_{n, j}, d_{n, j}\right]$, such that $\left|I_{n, j}\right|=\lambda_{n}$. We will also require that

$$
\begin{gathered}
I_{n, 2 j-1} \cup I_{n, 2 j} \subset \operatorname{Int}\left(I_{n-1, j}\right), \\
\frac{c_{n, 1}}{p_{n}} \in \mathbf{N}, \frac{c_{n, j+1}-d_{n, j}}{p_{n}} \in \mathbf{N} \text { for all } j \in\left\{1, \ldots, 2^{n}-1\right\},
\end{gathered}
$$

$$
c_{n, 2 j-1}-c_{n-1, j} \geq \frac{\lambda_{n-1}}{5}, c_{n, 2 j}-d_{n, 2 j-1} \geq \frac{\lambda_{n-1}}{5} \text { and } d_{n-1, j}-d_{n, 2 j} \geq \frac{\lambda_{n-1}}{5} .
$$

Let us define intervals $I_{n, j}$. Suppose that for a certain $n \in \mathbf{N}$ we have defined all $I_{n-1, j}$. Divide each $I_{n-1, j}$ into five intervals of equal length $\frac{\lambda_{n-1}}{5}$. If we choose the interval $I_{n, 2 j-1}$ inside the second one, and $I_{n, 2 j}$ inside the fourth one, then (9) and (11) clearly hold. Since by (6) and (8) $p_{n}+\lambda_{n}<\frac{\lambda_{n-1}}{5}$, it is easy to see that we can choose subsequently $I_{n, 1}, I_{n, 2}, \ldots, I_{n, 2^{n}}$ such that moreover $\left|I_{n, j}\right|=\lambda_{n}$ and (10) holds.

Put $K_{n}=I_{n, 1} \cup I_{n, 2} \cup \ldots \cup I_{n, 2^{n}}$ and $K=\bigcap_{n \geq 1} K_{n}$. Clearly $K$ is homeomorphic to the Cantor set.

Lemma 1. Let $n>2$ and $(z-h, z+h) \subset[0,1]$ be an interval such that $h \leq$ $(n-1) p_{n-1}$. Then $(z+h, z-h)$ intersects at most two components of $K_{n}$.

Proof. Thanks to (6) and (8) we have

$$
2 h \leq 2(n-1) p_{n-1}<\frac{1}{5} \sqrt{n-2} p_{n-2}=\frac{\lambda_{n-2}}{5} .
$$

So by (11) we obtain that $(z-h, z+h)$ intersects at most one component of $K_{n-1}$ and thus at most two components of $K_{n}$. 


\section{Construction of $T$}

Construction of $T$ is analogous to the construction in Lemma 2 from RodriguezPiazza [2].

Lemma 2. There exists a linear $T: C(K) \rightarrow C([0,1])$ such that for all $f \in C(K)$ :

(a) $T f(t)=f(t)$ for all $t \in K$.

(b) $|T f(t)| \leq\left(1-\frac{1}{2^{n}}\right)\|f\|_{\infty}$ for all $t \notin K_{n}$.

(c) Let $n>1$. If $I \subset[0,1]$ is an interval such that $I \cap K_{n}=\emptyset$, then $T f$ is Lipschitz on $I$ with the constant $\left(\frac{2\|f\|_{\infty}}{\frac{1}{5} \sqrt{n-1} p_{n-1}}\right)$.

Proof. For every $n \geq 0$ and every $j \in\left\{1, \ldots, 2^{n}\right\}$ pick a point $x_{n, j} \in I_{n, j} \cap K$. We define $T f(t)=f(t)$ for every $t \in K$. For every $n \geq 0$ and every $j \in\left\{1, \ldots, 2^{n}\right\}$, we define

$$
T f\left(c_{n, j}\right)=T f\left(d_{n, j}\right)=f\left(x_{n, j}\right)\left(1-\frac{1}{2^{n}}\right) .
$$

Extend $T f$ affinely on every interval $[a, b]$ where $f$ has been defined in points $a, b$ above and $f$ has not been defined in points of interval $(a, b)$ above. Conditions (a) and (b) are clearly fulfilled. It is easy to see that $F$ is a linear operator and it is obvious that $F f$ is a continuous function on $[0,1]$. Now verify (c). Clearly $|f(a)-f(b)| \leq 2\|f\|_{\infty}$ for endpoints $a, b$ of any interval $(a, b)$ on which $f$ has been defined affinely and (11) implies that every such interval which does not intersect $K_{n}$ has the length at least $\frac{1}{5} \sqrt{n-1} p_{n-1}=\frac{\lambda_{n-1}}{5}$.

\section{Construction of $F$}

Choose a sequence $\left\{y_{n}\right\}_{n=1}^{\infty}$ dense in $K$ and define functions $f_{n}$ :

$$
\begin{gathered}
f_{n}(t)=0 \text { for } t \in K_{n}, \\
f_{n}(t)=a_{n} \sin \left(\frac{2 \pi t}{p_{n}}\right) \text { for } t \in\left[0, c_{n, 1}\right], \\
f_{n}(t)=a_{n} \sin \left(\frac{2 \pi\left(t-d_{n, j}\right)}{p_{n}}\right) \text { for } t \in\left[d_{n, j}, c_{n, j+1}\right], \\
f_{n}(t)=a_{n} \sin \left(\frac{2 \pi\left(t-d_{n, 2^{n}}\right)}{p_{n}}\right) \text { for } t \in\left[d_{n, 2^{n}}, 1\right] .
\end{gathered}
$$

Notice that

$$
f_{n}\left(c_{n, 1}\right)=f_{n}\left(c_{n, j+1}\right)=0
$$

by (10). Put

$$
F f(t)=T f(t)+\sum_{n=1}^{\infty} f\left(y_{n}\right) f_{n}(t) .
$$

Thanks to (2) this series converges uniformly. Thanks to (2) and Lemma 2, F : $C(K) \rightarrow C([0,1])$ is a linear isometry so conditions (i) and (ii) from Proposition 2 are fulfilled. 
We will need the following simple fact.

Lemma 3. Let $I$ be an interval of length $p>0, M \subset I, 0<\alpha<1$ and $\beta \in \mathbf{R}$. For all $x, y \in M$ let

$$
\left|\sin \left(\frac{2 \pi x}{p}+\beta\right)-\sin \left(\frac{2 \pi y}{p}+\beta\right)\right| \leq \alpha .
$$

Then $|M| \leq \frac{3 p}{\pi} \arccos (1-\alpha)$.

Proof. Lemma 3 is proved in [1, Lemma 1] in the special case $\beta=0$. Applying this [1, Lemma 1] to the interval $I^{\star}=I-\frac{p \beta}{2 \pi}$ and the set $M^{\star}=M-\frac{p \beta}{2 \pi}$ we obtain our lemma.

Choose $z \in[0,1]$ and denote $\tilde{f}=F(f)$. We shall prove that, if $f \neq 0$, then for $\tilde{f}$ condition (iii) from Proposition 2 holds. It is enough to prove that

the set $S=\{x:|\tilde{f}(x)-\tilde{f}(z)| \leq \psi(|x-z|)\}$ has symmetric lower density 0 at $z$ for $z \in(0,1)$ and one-sided lower density 0 at points 0 and 1 .

Choose arbitrary $1>\delta>0$ and put $M=\left\{n:\left|f\left(y_{n}\right)\right|>\delta\|f\|_{\infty}\right\}$. If $f \neq 0$, then $\operatorname{card}(M)=\infty$. Choose an arbitrary $0<h<p_{1}$. Thanks to (7) there is a unique $n=n(h)$ such that $n p_{n}<h \leq(n-1) p_{n-1}$. First prove that

if $n \in M$ is big enough and $I \subset(z-h, z+h) \backslash K_{n}$ is an interval of length $p_{n}$,

$$
\text { then } \frac{|I \cap S|}{|I|}<\arccos \left(1-\frac{C}{n}\right) \text {, }
$$

where $C=4 \max \left(\frac{1}{\delta}, \frac{1}{\delta\|f\|_{\infty}}\right)$. Choose $x, y \in S \cap I$. The definition of $S$ and (4) give

$$
\begin{gathered}
|\tilde{f}(x)-\tilde{f}(y)| \leq|\tilde{f}(x)-\tilde{f}(z)|+|\tilde{f}(y)-\tilde{f}(z)| \leq \psi(|x-z|)+\psi(|y-z|) \\
\leq 2 \psi\left((n-1) p_{n-1}\right) \leq \frac{a_{n}}{n}
\end{gathered}
$$

Put $s_{n}(x)=\sum_{j=1}^{n} f\left(y_{j}\right) f_{j}(x)$ and $r_{n}(x)=\sum_{j=n+1}^{\infty} f\left(y_{j}\right) f_{j}(x)$. From (1) and (4) we have

$$
\begin{gathered}
\left|s_{n-1}(x)-s_{n-1}(y)\right| \leq|x-y| \sup _{t \in(x, y)}\left|\left(s_{n-1}^{\prime}\right)(t)\right| \\
\leq 2 \pi|x-y|\|f\|_{\infty} \sum_{i=1}^{n-1} \frac{a_{i}}{p_{i}} \leq 2 \pi p_{n}\|f\|_{\infty} \sum_{i=1}^{n-1} \frac{a_{i}}{p_{i}} \leq \frac{a_{n}}{n}\|f\|_{\infty} \text { and } \\
\left|r_{n}(x)-r_{n}(y)\right| \leq 2\|f\|_{\infty} \sum_{j=n+1}^{\infty} a_{j} \leq \frac{a_{n}}{n}\|f\|_{\infty} .
\end{gathered}
$$


Now from Lemma 2 (c) and (6) we obtain

$$
|T f(x)-T f(y)| \leq|x-y| \frac{2\|f\|_{\infty}}{\frac{1}{5} \sqrt{n-1} p_{n-1}} \leq p_{n} \frac{2\|f\|_{\infty}}{\frac{1}{5} \sqrt{n-1} p_{n-1}} \leq a_{n} \frac{\|f\|_{\infty}}{n} .
$$

Since $f\left(y_{n}\right) f_{n}=\tilde{f}-r_{n}-s_{n-1}-T f$, we obtain

$$
\begin{gathered}
\left|\sin \left(\frac{2 \pi x}{p_{n}}+\beta\right)-\sin \left(\frac{2 \pi y}{p_{n}}+\beta\right)\right|=\frac{1}{a_{n}\left|f\left(y_{n}\right)\right|}\left|f\left(y_{n}\right) f_{n}(x)-f\left(y_{n}\right) f_{n}(y)\right| \\
\leq \frac{1}{a_{n}\left|f\left(y_{n}\right)\right|}\left(|\tilde{f}(x)-\tilde{f}(y)|+\left|s_{n-1}(x)-s_{n-1}(y)\right|\right. \\
\left.\quad+\left|r_{n}(x)-r_{n}(y)\right|+|T f(x)-T f(y)|\right) \\
\leq \frac{1}{\delta\|f\|_{\infty} n}+\frac{3\|f\|_{\infty}}{\delta\|f\|_{\infty} n} \leq \frac{4}{n} \max \left(\frac{1}{\delta}, \frac{1}{\delta\|f\|_{\infty}}\right)=\frac{C}{n} .
\end{gathered}
$$

Thus Lemma 3 gives that

$$
|I \cap S| \leq \frac{3 p_{n}}{\pi} \arccos \left(1-\frac{C}{n}\right) \text { whenever } \frac{C}{n}<1 .
$$

So (13) really holds for $n$ big enough.

Choose arbitrary $z \in[0,1)$ and $h<1-z$. From Lemma 1 we get that $(z, z+h)$ intersects at most two components of $K_{n(h)}$ and $h>n(h) p_{n}$ so $\frac{\left|K_{n(h)} \cap(z, z+h)\right|}{h}$ $\rightarrow 0$. Clearly there exist $Z_{h} \subset(z, z+h),\left|Z_{h}\right|<3 p_{n}$ and $J_{1}, J_{2} \ldots J_{k} \subset(z, z+h)$ pairwise disjoint intervals of length $p_{n}$ such that

$$
(z, z+h) \backslash\left(K_{n} \cup Z_{h}\right)=\bigcup_{j=1}^{k} J_{j}
$$

Clearly $\frac{\left|Z_{h}\right|}{h} \rightarrow 0$ and for $J_{j}$ we can use (13). Since $\lim _{h \rightarrow 0+} n(h)=\infty$, we obtain $\arccos \left(1-\frac{C}{n(h)}\right) \rightarrow 0$. This all together gives us

$$
\lim _{\substack{h \rightarrow 0+\\ n(h) \in M}} \frac{|S \cap(z, z+h)|}{h}=0 .
$$

Analogously for $z \in(0,1]$ it holds that

$$
\lim _{\substack{h \rightarrow 0+\\ n(h) \in M}} \frac{|S \cap(z-h, z)|}{h}=0 .
$$

Since $\operatorname{card}(M)=\infty$, we obtain that (12) holds and we are done.

Proof of Proposition 3. If $f(t) \neq 0$ for all $t \in K$, then there exists $1>\delta>0$ such that $M=\mathbf{N}$. In this case our construction gives us that $\lim _{h \rightarrow 0+} \frac{|S \cap(z, z+h)|}{h}=0$ and $\lim _{h \rightarrow 0+} \frac{|S \cap(z-h, z)|}{h}=0$. So every point $z \in[0,1]$ is a Jarník point of $F f$.

For a given continuous function $f$ on $K$ find $c \in \mathbf{R}$ such that $f(t)+c \neq 0$ for all $t \in K$. Then $F(f+c)-c$ is the desired extension of $f$. 


\section{ACKNOWLEDGEMENT}

The author would like to thank to Professor L. Zajíček for proposing the problem treated here and for interesting discussions and valuable comments.

\section{REFERENCES}

1. J. Malý and L. Zajíček, Approximate differentiation: Jarnik points, Fund. Math. 140 (1991), 87-97. MR 92m:26006

2. L. Rodriguez-Piazza, Every separable Banach space is isometric to a space of continuous nowhere differentiable functions, Proc. Amer. Math. Soc. 123 (1995), 3649-3654. MR 96d:46007

3. A. Bruckner, Differentation of Real Functions, CRM Monograph Series, Volume 5 [2nd edition], Providence, Rhode Island, 1994. MR 94m:26001

Department of Mathematical Analysis, Charles University, Sokolovska 83, 18600 Prague 8, Czech Republic

E-mail address: Hencl@karlin.mff.cuni.cz 